\title{
A Design and Application of RFID Gantry
}

\author{
Quan Gang Wen ${ }^{1, a}$ Yongbing $X u^{2, b}$ \\ ${ }^{1,2}$ Computer Department Zhuhai College of Jilin University, Zhuhai China \\ awen_sir_125@163.com, bfengjiexyb@163.com
}

Keywords: Gantry RFID, Warehouse Management

\begin{abstract}
The warehouse management mainly consists of the processes of warehouse entry, warehouse delivery, storage moving and inventory verification. In traditional warehouse management, bar-code technology is widely used while the RFID becomes more and more popular in the modern warehouse management. By contrast, the RFID is more advantageous than the bar-code for its massive identification of goods in every aspect of a process in the modern warehouse management. Based on the requirements of the process of in-out stock, we have made an analysis on the principle of RFID and designed the device of RFID gantry which meets a standard of ISO/IEC 18000-6. The practical running of this device involves the working of both hardware and software. The testing result shows it can be used in practical logistical warehouse management and bring about better ends. The application of the RFID technology can promote the speed of in-out stock, consequently reducing spare stocks, improving efficiency in space utilization and keeping the cost of work force down.
\end{abstract}

\section{Introduction}

In the management of modern logistical warehouse, the RFID technology is more and more applied in the intelligent management of the storage goods. The typical process of warehouse management mainly consists of the processes of warehouse entry, warehouse delivery, storage moving and inventory verification. In traditional warehouse management, the bar-code technology is mostly adopted. By this technology, we have to scan goods one by one during every stages of warehouse entry, therefore slowing down the entry and wasting work force. But the warehouse management system that we have designed adopts the RFID technology as identification of goods, which meets the standard of ISO/IEC 18000-6. This kind of tags is a passive UHF and the issues of discrimination distance, rate and ratio need to be dealt with in the application of these tags. We should take an overall consideration with the factors like the power of the antenna, the position and direction of the tags, the collision of tags. In the warehouse entry we firstly unload the goods, and then bind a RFID tag to each goods, put these goods on a transfer trolley. After scanning the goods for mass discrimination through RFID gantry, we do the goods shelves process finally. To meet with the requirement of rapid warehouse entry and mass discrimination in this process, we have designed the RFID gantry.

This thesis consists of five chapters which are organized as follows. The architecture and function requirements of RFID gantry are discussed in the first place. Then we explain the design of crossing RFID gantry according to the practical requirements in the second part. We then analyses the key techniques of RFID gantry and its working process. In the fourth part function testing and performance testing of RFID gantry are introduced during the actual application. The thesis is concluded in the fifth part.

\section{Architecture and Functions of RFID Gantry}

\subsection{Architecture of RFID Gantry}

Gantry is generally composed of steel structure, transmission system, electrical system and safety control system. The gantry we have designed is made primarily for the installation of the RFID reader, which is as simplified as a kind of portal frame and light steel structure. This structure has the 
characteristics of effective material using, small-sized and light-weight. It can be massively produced in the factory with the quality guaranteed.The structure of RFID gantry as shown in Figure 1.

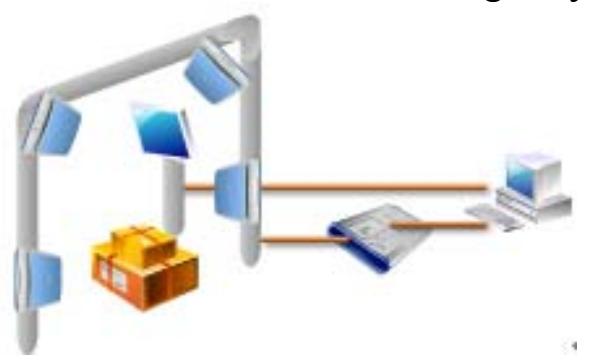

Figure 1: Essential Structure of RFID Gantry

A system of RFID reader system is deployed in this steel structure, which is composed of the following:

Two four-channel readers: they are connected to computer through a router and the IP address is configured in the same segment.

Four circularly polarized antennas: They are installed in pairs. Two of them are deployed right in front of the main passageway of goods which is about one meter high from bottom of the gantry to these antennas. The other two antennas are deployed inclining to the passageway of goods with the height of about two meters from bottom of the gantry to these antennas

One main control computer: The software of the RFID reader is installed in main control computer. The control computer communicates with server via local area network.

One display screen: The reading and writing information are shown in this screen.

\subsection{The Requirements of RFID Gantry.}

The basic process of warehouse entry is as follows: when the cargo is arrived at warehouse, workers distribute a RFID tag to each item of goods according to the quantities in the cargo list. Then these tags are bound to the goods that are transported by a small cart. All the goods are identified and the quantities are counted automatically when the carts go through the RFID gantry as the completion of the warehouse delivery. In this actual process, the max number of goods that a small cart can transport each time is up to 500 items. It will take 5 seconds for a cart to go through the RFID gantry at normal velocity.

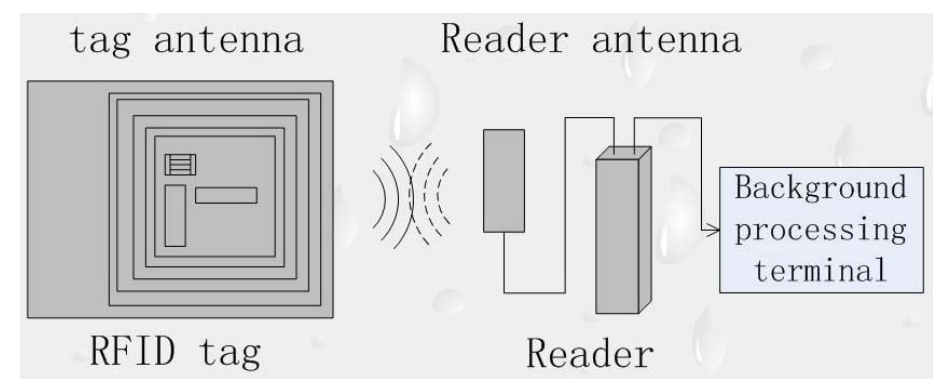

Figure 2: Reading and Writing Principle of RFID Tag

The reading and writing principle of RFID tags is as shown in Figure 2. The antenna of RFID reader can send and receive a certain radio frequency (RF) which generates the magnetic flux. The RFID tag antenna receives the energy which drives the tag's inner circuit and reflects its label information to the RFID reader by the reflection of radio frequency. Thus, the RFID reader can identify a tag. In our actual application, some requirements are demanded in the distance reading of a RFID, the direction of a RFID tag, the recognition speed and ratio etc. Hence, the following discussion is concerned.

\section{Recognition Distance}

The reading or writing distance of RFID tags are determined by the factors of the power, the size and position of the RFID reader antenna. The device CSL461 we use is a four-port fixed UHF RFID reader which meets EPCClass1Gen2 standards with accordance to ISO/IEC 18000-6 protocol, and it can read or write RFID tags of UHF band at about $860 \sim 960 \mathrm{MHz}$ with the max communication distance of about 10 meters[2]. Actually, the distance between the RFID reader and tags should be 
limited within no more than 1.5 meters as we only require to scan tags of goods on the small cart when they are passing through the RFID gantry. Longer communication distance will bring about side-effects, that is, those tags we don't want can also get scanned 1.5 meters away. To avoid this we can install a metal shell to block it. But the best way to deal with it is to adjust the power of the RFID reader to control the reading distance .

From the analysis of the working process of passive UHF tags, we know its energy transfer includes forward link transmission and the reverse link transmission[1]. The forward link transmission means the energy is transferred from RFID reader to tags. Suppose the power density is $\mathrm{S}$, the distance from RFID reader is $\mathrm{R}$, we can define it as flowing:

$$
S=\frac{P_{t} G_{t}}{4 \pi R^{2}}=\frac{P_{E I R P}}{4 \pi R^{2}}
$$

In this formula, $\mathrm{P}_{\mathrm{t}}$ represents transmission power of RFID reader; Gt represents gainning of RFID reader antenna; $\mathrm{P}_{\text {EIRP }}$ represents effective radiated power of RFID reader. Hypothetically, when the angles of magnetic lines and the electronic tag is right angle, it is the best, and when the biggest power $\mathrm{P}_{\mathrm{tag}}$ which the tag receives is proportional to $\mathrm{S}$, it can be defined as following:

$$
P_{\text {tag }}=A_{e_{-} \text {tag }} S=\frac{\lambda^{2}}{4 \pi} G_{\text {tag }} S=P_{\text {EIRP }} G_{\text {tag }}\left(\frac{\lambda}{4 \pi R}\right)^{2}
$$

In this formula, $A_{e_{-} \text {tag }}$ represents the effective area of RFID reader antenna; $G_{\text {tag }}$ represents effective radiated power of RFID reader antenna.

The reverse link transmission means the energy is reflected from tags to RFID reader. The reflected energy is proportional to cross section of the radar. The reverse energy from tags to RFID reader $\mathrm{P}_{\mathrm{r}}$ is defined as following:

$$
P_{\mathrm{r}}=A_{e_{-} \text {reader }} S_{\text {back }}
$$

When RFID reader's sending and receiving share the same antenna, the effective area of RFID reader antenna is defined as following:

$$
A_{e_{-} \text {reader }}=\frac{\lambda^{2}}{4 \pi} G_{\mathrm{t}}
$$

The radiation power density $S_{\text {back }}$ is generated when the electromagnetic wave reflects from tags to RFID reader which is defined as following:

$$
S_{\text {back }}=\frac{P_{\mathrm{t}} G_{\mathrm{t}} \sigma}{(4 \pi)^{2} R^{4}}
$$

Put $\mathrm{A}_{\mathrm{e}-\text { reader }}$ and $\mathrm{S}_{\mathrm{back}}$ into the formula of $\mathrm{Pr}$ and it is as the following:

$$
\mathrm{P}_{\mathrm{r}}=\frac{P_{\mathrm{t}} G_{t}{ }^{2} \lambda^{2} \sigma}{(4 \pi)^{3} R^{4}}
$$

Based on the analysis above, we realize the relation between RFID reader's transmit power and RFID read-write distance, which we can apply to adjust the reader's transmit power to meet with suitable scan distance.

\section{Direction of Tags}

The energy of passive RFID is provided by magnetic coupling between RFID reader antenna and tags. So the direction of tags directly influences the coupling coefficient which has great impact on energy gaining and the reliability of communication. We use Double antenna in the design of RFID gantry. The simulation diagram of magnetic field distribution about double antenna is shown in Figure 3[3]. 


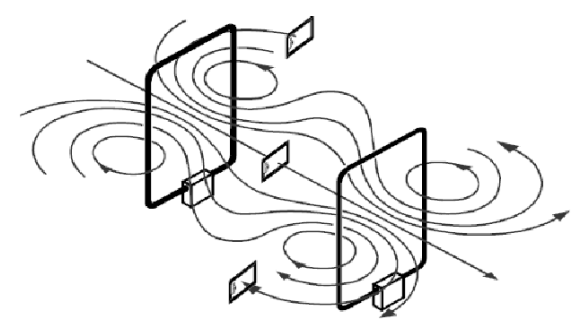

Figure 3 Simulation Diagram of Magnetic Field Distribution About Double Antenna

\section{Requirements of Recognition Rate And Reading Speed}

In actual application, the cart allows up to 2 times back and forth of going through the RFID gantry. During the first entry, recognition rate is required up to $98 \%$ and the second is then required to $100 \%$. The requirement of read speed is as follows: The max number of the pieces of the goods in one cart is 500 at a time. It will take 5 minutes to go through the gantry at a normal speed. As a result, the minimum reading speed is of 100 tags a second. By contrast between different devices, 2 UHF

(920-925Mhz) RFID readers by Impinj company are used in our project. The RFID reader of this type has very high recognition rate, reading speed and really high density read-write mode.

\section{Control and Implementation of Crossing RFID Reader}

On the side of hardware, two RFID readers are connected to the control host through the router. The major function of the reader is to monitor on programming suitable software to realize the function of automatic identification goods when passing the gantry. The control procedure of the gantry is as follows:

Check local configure files. If there are local configure files, they are loaded; otherwise, the program is exited and a message is given to the user to configure the RFID reader.

Initialize RFID reader. if it is achieved, the configure file is applied to the RFID reader. The successful application means the reader is connected; otherwise, the program is exited.

Set scan parameters. The reader object calls the Inventory function which will return a value. According to this value, the start of the scanning is confirmed and the user is notified.

Receive scan data. A string type Array tags[] is used to store the return data, if a data is in this array, the program is exited. Otherwise, the data is added to array tags[] which will be send to the user interface of application program. It is shown as figure 4.

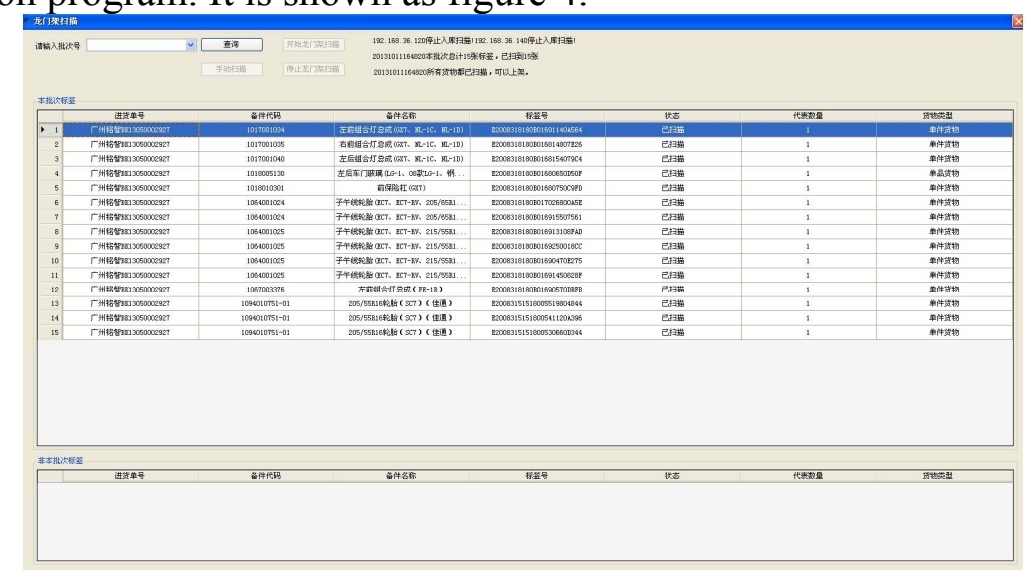

Figure 4: scan function of RFID gantry

\section{Testing and Application of RFID Gantry}

\subsection{Function Testing.}

According to the analysis of design above, we deploy a complete REID gantry into a logistical warehouse which is divided into A-E five zones. The goods in these five zones are classified into three categories: 
Single type goods: This type of goods is stored into zone B,C,D,E with the forms of carton or wooden packaging.

Single class bulk goods: It has an appearance of a large box which contains several one single class good.

Multi-class large package goods: it has an appearance of a large package of several multi-class goods. In general, these types are mainly stored in A zone, another few are placed in other zones.

Before adopting RFID technology, bar-code technology is used in this warehouse. After using RFID, the process of the goods management is shown in Figure 5.

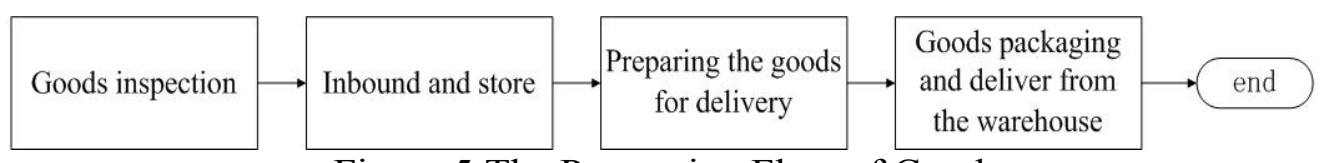

Figure 5:The Processing Flow of Goods

Goods inspection: For single type goods, appearance damage and quantity of the goods are checked according to the incoming packing list. In present there are two workers in charge of this process.

Inbound and store: After goods inspection, workers take the goods by small cart and put them into storage unit. If bar-code is used, only one piece of goods is scanned at a time. It will require four workers to do this job. But we only need only one worker if adopting RFID technology.

Preparing the goods for delivery: Workers will prepare the goods according to the bill of sales. The system will automatically arrange the sequence of delivery from the maximum quantity storage shelf to the minimal with RFID handheld devices. In this procedure, workers can be reduced from four to three.

Goods packaging and deliver from the warehouse: According to the bill of sales, all of the goods are packaged. In this process there need four workers if using bar-code system. But with RFID system, only two workers are needed.

In the processes introduced above, RFID gantry is used in inbound, stock taking and outbound. The data has shown that the RFID system can handle the logistic for the warehouse. In 1000 times of scanning, 989 times are 100\% scanned in the first entry of RFID gantry. Only 9 times of entry need rescanning to recognize all RFID tags. But there are 2 times of entry which can not be recognized anyway. The reason is that tags on the goods are damaged. In summary, our design of RFID gantry system can get a $98.9 \%$ of successful rate at one turn, and a $99.8 \%$ successful rate for second turn, which clearly shown that this system can meet the requirements of logistic warehouse. With the application of our system, the number of workers in the warehouse can be reduced from fourteen to eight. In theory the efficiency are increased at $42.9 \%$.

\subsection{Performance Testing}

In the case of meeting above functions and requirements, two factors are closely related with the usability of the system. They are the peak value and the distance of tags recognition in a dense tags case. The cart we use in this system can carry up to 500 pieces in a time when going through RFID gantry. When going through RFID gantry, two readers are used to poll scanning. By analyzing scanning data, the relationship between tags numbers and time can be shown in Figure 6 . The peak value is $998 \mathrm{tags} / \mathrm{s}$.

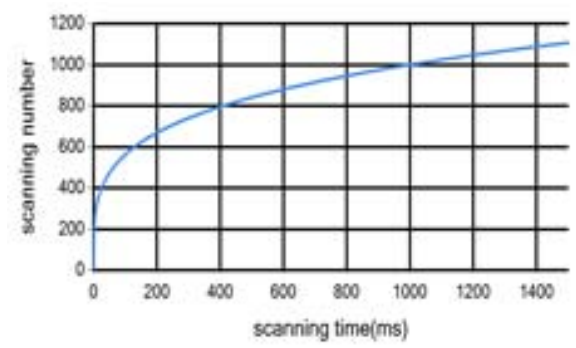

Figure 6 The Relationship Between Tags Numbers and Time 
In fact, the distance of scanning should be limited for the accuracy of the data. Longer scanning radius will get the outside RFID tags scanned. So it will cost the extra working of discriminating redundancy tags, and efficiency of the system will be declined. For this reason, adjustment of antenna' $\mathrm{s}$ emission power is made to meet the requirement of suitable scanning radius[4]. Real testing data of the relationship between antenna' s emission power and scanning radius is shown as figure 7 .

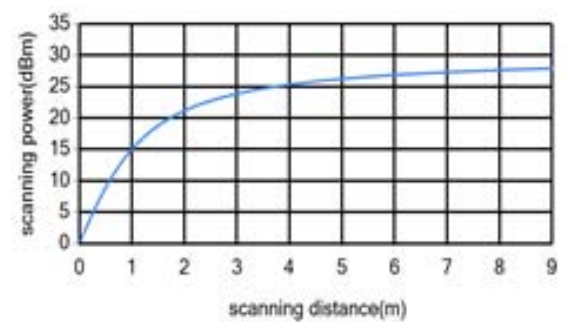

Figure 7The Relationship Between Emission Power and Distance

The data shows that the relationship between antenna' $\mathrm{s}$ emission power and scanning radius is consistent with the theory in section 2.2. The suitable emission power is about $18 \mathrm{dbm}$, which meet the distance of 1.5 meters.

\section{Conclusion}

In this thesis, we have analyzed requirements of all aspects of warehouse management systems and provided a RFID gantry design. Combined with relevant principles of RFID technology, the hardware and software have guaranteed completion of this RFID gantry. With the practical application of the device, function testing and performance testing are carried out. The analysis of testing data of warehouse management system shows that a first turn of recognition rates to $98.9 \%$, the secondary turn of recognition rates to $99.8 \%$. This result meets usability of this system. By contrast with bar-code technology, the using of RFID technology bring about the following advantages: The speed of goods inspection is increased by $10 \%$; Inventory is reduced by $10 \%-30 \%$; Inventory space utilization can be increased by $20 \%$; The overall efficiency is increased by $42.9 \%$; The cost of workforce is decreased by $10 \%-40 \%[5]$.

\section{References}

[1] TANG Zhijun, Research on antennas and Propagation characteristics in Passive back scatter RFID systems, doctoral dissertation,05.2010

[2] http://solution.rfidworld.com.cn/2013_09/eff3acc2697aaala.html

[3] Zhang Guiyan, The Research of long-range RFID Reader Based on ISO15693,master's thesis, 01.2010

[4] Huang Guo wei,The Study about a Controlling Method of RFID Communication Distance,master's thesis, 12.2009

[5]Implementation Plan of RFID Warehouse Project,Guangzhou Zhongda Microelectronics, Inc.

, 08.2012 